\title{
A REVIEW ON MOTION TRACKING ALGORITHMS
}

Farah Jamal Ansari ${ }^{1}$

Abstract- The Main motive of this paper is to put forward a comprehensive research review in the domain of Motion tracking algorithm in real time. the main focus is on the tracking algorithms and its application. More than 50 research paper on the motion tracking domain are studied and research works are acknowledged with proper references in order to exemplify the important issues and their relations to the various methods.

Keywords - Kalman Filter, Object tracking, kernel tracking, Dynamic motion tracking

\section{INTRODUCTION}

Video surveillance system used to monitor areas sensitive to long safety. Finding videos, and providing useful information can be obtained in advance. Detection and tracking forms a most important usage in computer vision such as video observation, vision depends control, human-computer interfaces, medical picturing, augmented reality, and robotics. About moving object detection from an object control is a big step forward. Segmentation of objects, in a static environment is not complicated. In relation to dynamic changes in environmental conditions: wind, segmentation of objects, light, shade and branches of whispering trees should be treated as a powerful monitoring system is a difficult and important problem. Object tracking is to track an object (or multiple objects) over a order of picture. The segmentation of the object of interest of a video of the object and its movement, orientation, etc. The occlusion is defined as the course of the track in order to obtain useful information. Against the motion the problems that arise with the track of sharp objects, the object is changing, too, and the event is not to act against a hard object structures, object-to-scene Occlusions and movement of the camera. Tracking is usually performed in the context of higher- level applications that need the position and/or shape of the object in every frame. The analysis of objects in real time monitoring important exposure in the field of development and is more and more recently. Motion detection and object tracking method for the prevention of threats against the video surveillance system can also be used.

Each tracking method or an object in each frame requires a mechanism to determine the formation of the first film or object. As part of an object in order to determine the general approach is the use of the data. However, some object detection, count a number of methods to reduce the number of false detections of the temporary use of information.

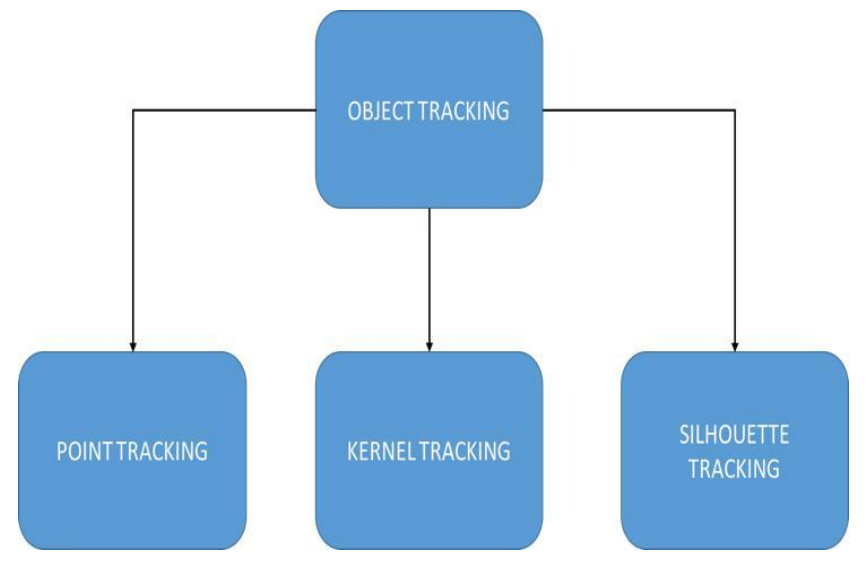

Figure1: Object tracking method

This temporal information is normally in the form of frame changing, which highlights changing area in successive frames. Tracking includes registering the actions of the segmented thing from starting frame to the end frame in a video.

\footnotetext{
${ }^{1}$ Department of Computer Sciences, University Polytechnic, Jamia Millia Islamia, New Delhi-110025
} 


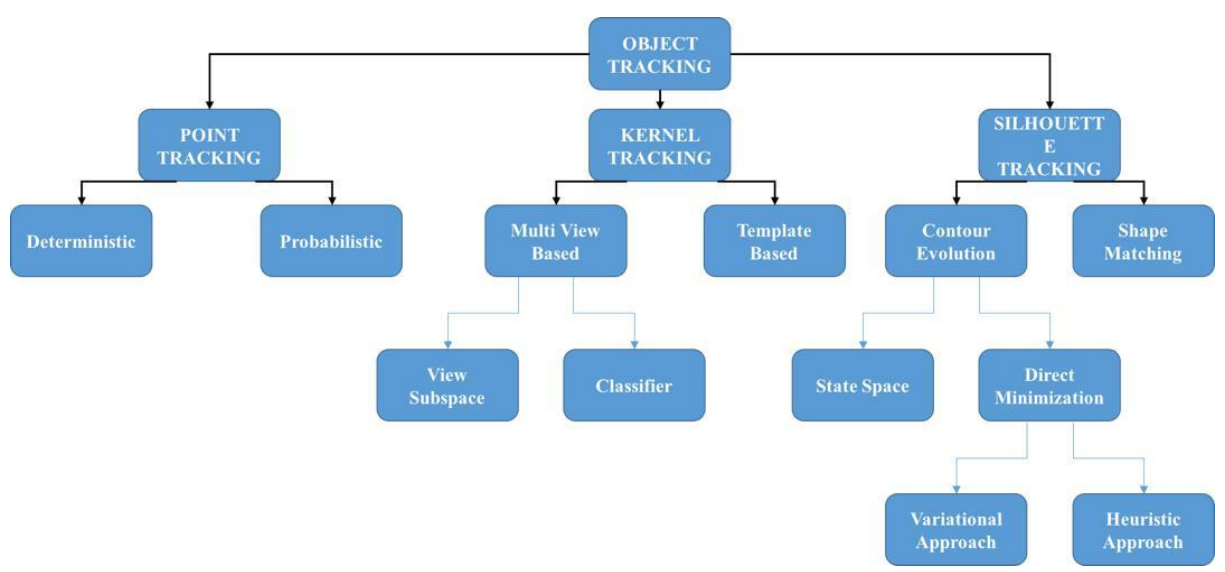

Figure 2: Classification of tracking methods

\section{REVIEW OF LITERATURE}

Children safety has always been a critical issue. The casualties of children may increase dramatically when or guardians are absent. Therefore, the image of the parent video-based monitoring system can be praised for caring for children. In this paper, based on the double camera system monitoring the circulation of technology and the manufacturing system works on the basis of this proposal, is coming. The system can observe and follow the children's condition, solve the danger state of the children and make proper reaction to the dangers, i.e. by triggering the alarm and starting something essential to avoid the bad significance. The OpenCV library for image and video processing, and children's behavior and for information about location and status. The experimental results in the recognition of a control system for the basic movements of children and the basis of rules that can take the necessary measures. Due to its advantages of low-cost in hardware and fast response the monitoring system can be implemented as an additional. [1]

We identify with the protection of the visual person, and a more autonomous robot, the algorithm for the novel. People swimming domain patterns of frequency and opportunities with the use of visible space security between domains, we will develop an algorithm to determine the position and orientation of the diver. Our algorithm is very different in the way that it permits detection of arbitrary motion directions, in addition to keeping track of a diver's position through the image sequence over time. A hidden Markov model (HMM) approach based on the search of images in the spatial domain, which will rely on all the trajectories of space erases. Diver accurate determination of the frequency domain situation and allows you to determine the direction of movement, but the passage of the crop to avoid the geometric search of large spaces and provides for efficient computing. Experimental validation of the proposed approach is presented on datasets collected from open-water and closed water environments. [2]monitor the movement of the human body in real-time synthetic environments, robots and other important applications of human computer interaction technology. This document is targeted to wing segments in real time has been developed to evaluate Kalman Goals extended filter. small inertial modules of sensors containing triaxial angular velocity sensor, data processing and magnetometer accelerometers. filter instead of the Euler angles or axis / angle pair is what it is through the group. Preprocessing of the acceleration and magnetometer measurements using the Quest algorithm produces a computed quaternion input for the filter. This preprocessing reduces the size of the state vector and the measurement of linear equations. Real-time implementation and testing results of the quaternion-based Kalman filter are presented. Check the results of the experimental design of the filter, as well as the development of the human body moving in real time / access to the magnetic sensor modules. [3]

We present and the control movement used in recognition of kanji sports signals will present two case studies. Through them, the analysis based on the camera, and portable devices to observe the movement - how to get through them, we will sign two main methods can be adopted in the example of sports applications. In the first case, advanced video frame analysis is applied to gesture recognition for improved fencing training. In the second case, Kanji used to track the movement of portable gaming sports and attack recognition. Two sample recognized prototype will be carried out on various platforms, and not used in practice. [4]

In this work, several main cameras, binoculars for grayscale images sequences of interior environment provides the basis for the tracking of human movement. Gaussian models in many different places are taken by a camera installed in a constant between frames used for human subjects. The results of the experimental data and the robustness of the algorithm show that the potential for applications in real time. [5]

The Bayesian estimation of 3D human motion from video orders is quantitatively evaluated using synchronized, multicamera, calibrated video and 3D ground truth poses learned with a commercial motion capture system. While many methods for human pose estimation and tracking have been proposed, to date there has been no quantitative comparison. Our main motive is to evaluate how different pattern select influence tracking performance. Toward that last, we independently implemented two fairly standard Bayesian person trackers using two alternatives of particle filtering and suggest an evaluation measure suitable for assessing the quality of probabilistic tracking techniques. In the Bayesian framework, we 
match different picture likely hood functions and prior models of human motion that have been projected in the literature. Our results suggest that in constrained laboratory environments, present technique perform quite good. Multiple cameras and background subtraction, however, are essential to attain reliable tracking signifying that many present techniques may be inappropriate in more normal settings. We discuss the implications of the study and the directions for future research that it entails. [6]

During the last two years in the use of technology, art projects, artistic performances and structures for use as the projections of the movement of mutual activity is increasing. However, this interaction is responsible for the creation of the artists are based on the application of this type of system, many computer experts. The tool herein presented, Motion Designer, intends to assist the design of these systems by providing artists with higher levels of autonomy and efficiency during the creative process, allowing them to specify the rules by which a human body interacts with both the audio and the visuals used in their interactive art work. As provided to the interpreter to modulate body movement is based on the RGB-D camera. Artists will be modified in order to take Action Designer. weapon-tested with dancers, ballet masters and architects. Results Motion Designer works as a catalyst for your creativity, artists, shows that for the valuable assistance. [7]

It is recommended that it represents the objective and towards a new form of reasonable restrictions in the central part of object observation. Target entity based on histogram representation is regulated by hiding in a space of the isotropic core. The masking induces spatially-smooth similarity functions suitable for gradient-based optimization, hence, the target localization problem can be formulated using the basin of attraction of the local maxima. We use Bhattacharyya, as a measure of the measure of similarity, and optimize the performance of the control process. In the examples given in the new method was successfully camera movement, partial occlusions, scale, disorder and destination variations. A combination of filters and information, association and movement methods is discussed. We describe a couple of applications that you can only use: basic information, the Kalman.Goals movement tracked by the face of a model and control. [8]

An unknown environment of mobile devices with the command followed by locations, in this document, presents an architecture combining a monocular camera and an inertial measurement unit (IMU) in ubiquitous mobile devices. IMU acceleration module and angular velocity with high frequency, but the inclusion of the group based on the movement is much closer to the drift down. According to the vision, motion tracking, but will ensure maximum precision, but can work with scenes texture environment weak or dynamic. The IMU and a binocular camera, this paper on the proposed approach will be combined with extended state error-based Kalman filter pair. With the combination of the advantages the monocular camera and IMU, The proposed method for mobile devices with limited resources in real time for the achievement of this movement. Finally, the proposed motion is measured using the method of the follow-up experiments. [9]

In this work, unbalance measurements of the markers attached to the body to control the movement of the human body based on the novel online. And for this reason, we have attached markers, including location, use of a kinematic model of the human body. Based on the model, we have the basis of a limited filtering of the sample and multi-target tracking methods would be used in combination: 1) the constraints imposed by the human body articulation from time to time based on the objectives of the world through the introduction of a single parameter, and 2) nearest neighbor algorithm (GNN) markers and first counting measurements for one-to-one communication, and 3) a pair of snake eyes only (MHT), which allows the user to send strong demand. The rapid and accurate evaluation proposed for complex real-time applications, allowing a very precise position. In addition, markers, and provide robustness to disorders of partial occlusion, as well as the inevitable measure. [10] In this work, we have the treatment for the purposes of high precision measurement system and Microsoft TM cannot compare the performance of V2 models. A movement is accurate and low cost of the system is important for the development of the treatment of daily living therapeutic assistance conditions. Upcoming therapeutic assistance systems have to be affordable and able to analyze the motion of the inhabitant. Therefore, the low evaluation v2 sensor models to measure the sensor angle sensor of the board should be considered. Compared to high precision measurement up to $8.4^{\circ}$ median deviation could be achieved. We will v2 sensor allows the exercise compatibility analysis for the treatment of conditions, we can conclude that. [11]

Microsoft, for example, can grow as low-cost depth sensors, have potential for non-contact health monitoring that is strong to ambient lighting situations. However, the depth of the captured images often suffer from a high noise and, therefore, to develop a difficult biometric to evaluate them. In this paper, we suggest to capture deepness video of a human subject with the help of Kinect 2.0 to estimation his or her heart rate and rhythm; defined for the analysis of the frequency of blood flow due to mechanical movement through the head from the heart and is pumped to the head was determined to make a small movement. Specifically, we 1st reestablish a taken depth video via a joint bit-depth improvement technique, with the help of a graph-signal softness previous for regularization. $2^{\text {nd }}$ one is, 3D motion vectors are derived for depth video found in the head area. The spotted vectors are fed back to the wisdom restoration methods in a itrrention to confirm that the motion information in 2 methods are reliable, enhencing performance of both restoration and motion following. $3^{\text {rd }}$ one is, the calculated 3D motion vectors are projected onto its main element for one dimmensional signal examination, collected of trend elimination, band-pass filtering, and wavelet based motion denoising. Finally, the heart rate measured by the Welsh power spectrum analysis calculates by determining the upper part of the heart rate. For the results of an experimental portable oximeter with the discount rate and the rhythm of our proposed algorithm provides the heart rate and the precise rhythm. [12] 
a new encoding standard video MPEG-4 encoding and content-based services, in the form of products, as well as allows you to move data, taking into account, as well as high efficiency. the planes of video objects (VOP) for the objects in video sequences and the segmentation of the new mining algorithm in motion is proposed in this article. An exposure with a number of objects within the components added for analysis and the subsequent VO is based on language-specific extraction of video objects (VO) are discussed. Our algorithm begins with a robust double-edge map derived from the difference between two successive frames. After removing edge points which belong to the previous frame, the remaining edge map, moving edge (ME), is used to extract the VOP. The proposed algorithm, as well as low-end cameras, MPEG-4 test sequences were closed and evaluated on the basis of promising results. [13]

This paper describes an end-to-end method for extracting moving targets from a real-time video stream, classifying them into predefined categories according to image based properties, and then robustly tracking them. By moving the recognized target by the difference between the smart photo-pixel. Measured classified a temporal sequence of these functions is restricted and classified into three categories: people, teams or background after the disturbances in secrecy, inclusion and temporal human, vehicle or background clutter Once classified, targets are tracked by a combination of temporal differencing and template matching. The system defines the objectives, the main ways to refuse cluttel; and changes in the appearance of the occlusions, and the goal of stopping movement, despite the great distance and time. [14]

On an object based on color image information in a simple and very fast method development. Running Work with a Silicon Graphics Indy R4600 IndyCam system our time frame size algorithm (640 x 480 pixels), and video frame rate (30 hours), according to the tracking objects. Robustness with respect to occlusion is attained via an explicit hypothesis-tree demo of the occlusion methods. We reveals the capicity of our methods in the challenging task of following people, particularly following human heads and hands. [15]

For multi-object tracking in aerial videos which are acquired from moving cameras, the motion of cars is complicated by global camera movements and always unpredictable. To arrangement with such unpredicted camera gesture for online multivehicles tracking, structural motion context between objects has been used thanks to its robustness to camera motion. In this paper, we propose an effective data association method that exploits structural motion context in the existence of big camera motion. In adding, to further enhance the robustness of algorithm against missing data due to the target being occluded behind other objects, an appearance context model is developed to represent appearance information of objects we need to track. The structural motion context and appearance context are then used to predict the location of the unobserved objects. Experimental results on VIVID datasets show the effectiveness of the proposed algorithm for multi-object tracking. [16] In this work, we briefly summarize our video surveillance and the basis of the investigation. We then examination persent research on Human action recognition, and our persent work on real-time multi-person following. By implimenting adaptive background subtraction technique, foreground area are $1^{\text {st }}$ we recognized and segmented. A clustering technique is then used to group the foreground pixels in an unsubstantiated means to approximation the picture position of specific persons. A Kalman filter is implimented to keep flow of individually human and a unique label is allocated to each followed singal. Depends on this method, people can enter and go the scene at random. Abnormity, such as silhouette merging, is handled elegantly \& single persons can be flow appropriately after a group of people divided. Experiments establish the real-time output and robustness of our system working in complex scenes. [17]

The camera is based on body identification and human control is widely used in the field of military and civilian vision computer is under study. In this paper, we present an improved algorithm of human body tracking depends on better camshaft algorithm. Firstly, we have some common image noise reduction algorithms. By combination the frame modification and background removal technique, an enhanced moving target recognition algorithm is sugested, by which the completely area of target can be found. Then, with the analysis of particle filtering and traditional Cam-shift algorithm, we added a letest human body tracking technique that is able to choose the target automatically due to the detection output. Identification and evaluation of the results of the motion parameter control algorithm is analyzed. Finally, the identification of the human body and to experience a series of recommendations aimed at demonstrating the efficiency of the algorithms. [18]

\begin{tabular}{|l|l|l|l|}
\hline S.No. & \multicolumn{1}{|c|}{ Author } & \multicolumn{1}{|c|}{ Topic } & \multicolumn{1}{|c|}{ Result } \\
\hline 1. & Weiyang Zhang & $\begin{array}{l}\text { Design of a Dual Camera Children } \\
\text { Monitoring } \\
\text { System based on Motion Tracking } \\
\text { Technology }\end{array}$ & $\begin{array}{l}\text { this system can judge the situation and send } \\
\text { messages } \\
\text { when children in danger. }\end{array}$ \\
\hline 2. & $\begin{array}{l}\text { Md Jahidul Islam } \\
(2017)\end{array}$ & $\begin{array}{l}\text { Mixed-domain Biological Motion Tracking } \\
\text { for Underwater Human-Robot Interaction }\end{array}$ & $\begin{array}{l}\text { This shows, we have positive detection } \\
\text { accuracy about } \\
84.2-91.7 \% \text { over all cases. }\end{array}$ \\
\hline 3. & $\begin{array}{l}\text { Xiaoping Yun } \\
(2006)\end{array}$ & $\begin{array}{l}\text { Design, Implementation, and Experimental } \\
\text { Results of auaternion-Based Kalman Filter } \\
\text { for Human Body Motion Tracking }\end{array}$ & $\begin{array}{l}\text { The avatar was able to successfully track the } \\
\text { human } \\
\text { arm motion in real time under all conditions. }\end{array}$ \\
\hline 4. & $\begin{array}{l}\text { Reneta P. Barneva } \\
\text { (2016) }\end{array}$ & $\begin{array}{l}\text { MOTION TRACKING FOR GESTURE } \\
\text { ANALYSIS IN SPORTS }\end{array}$ & $\begin{array}{l}\text { The two different } \\
\text { types of motion tracking for gesture }\end{array}$ \\
\hline
\end{tabular}




\begin{tabular}{|c|c|c|c|}
\hline & & & $\begin{array}{l}\text { recognition in the } \\
\text { context of sports applications. }\end{array}$ \\
\hline 5. & $\begin{array}{l}\text { Q. Cai and J. K. } \\
\text { Aggarwal (1996) }\end{array}$ & $\begin{array}{l}\text { Tracking Human Motion Using Multiple } \\
\text { Cameras }\end{array}$ & $\begin{array}{l}\text { Multi fixed cameras: } \mathrm{Sum}=0.86 \text { in } 3.0 \text { Single } \\
\text { cameras:Sum }=1.37 \text { in } 1.82\end{array}$ \\
\hline 6. & $\begin{array}{l}\text { Filipe Baptista } \\
(2016)\end{array}$ & $\begin{array}{l}\text { Motion Designer: Augmented Artistic } \\
\text { Performances with Kinect-Based Human } \\
\text { Body } \\
\text { Motion Tracking }\end{array}$ & $\begin{array}{l}\text { Motion Designer is a valuable aid to artists, } \\
\text { working as } \\
\text { a catalyst of their creative process. }\end{array}$ \\
\hline 7. & $\begin{array}{l}\text { Dorin Comaniciu } \\
(2003)\end{array}$ & Kernel-Based Object Tracking & $\begin{array}{l}\text { The kernel-based tracking technique, when } \\
\text { combined } \\
\text { with prior task specific information, can } \\
\text { achieve } \\
\text { reliable performance. }\end{array}$ \\
\hline 8. & $\begin{array}{l}\text { Huanjun Deng } \\
\text { (2016) }\end{array}$ & $\begin{array}{l}\text { A motion tracking technique by joining the } \\
\text { IMU and camera in mobile devices. }\end{array}$ & $\begin{array}{l}\text { Achieve real-time ego-motion estimation in } \\
\text { resource- } \\
\text { constrained mobile devices and our motion } \\
\text { tracking } \\
\text { system can run } \\
\text { in real time (about } 25 \mathrm{fps} \text { ). }\end{array}$ \\
\hline 9. & $\begin{array}{l}\text { Cheng } \\
(2017)\end{array}$ & $\begin{array}{l}\text { Estimating Heart Rate and Rhythm via 3D } \\
\text { Motion Tracking in Depth Video }\end{array}$ & $\begin{array}{l}\text { show accurate estimation of the heart rate and } \\
\text { rhythm } \\
\text { using } \\
\text { our proposed algorithm as compared to rate } \\
\text { and } \\
\text { rhythm } \\
\text { estimated by a portable oximeter. }\end{array}$ \\
\hline 10. & $\begin{array}{l}\text { Changick } \\
\text { Kim(2002) }\end{array}$ & 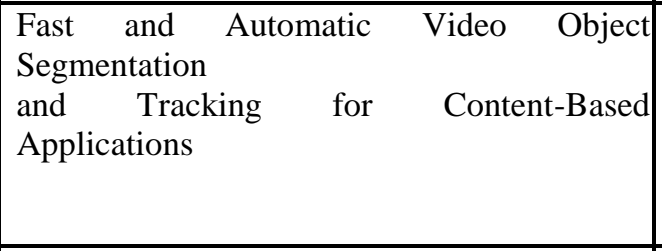 & $\begin{array}{l}\text { results generated from on-line single object } \\
\text { segmentation and tracking system, where } \\
\text { only a face } \\
\text { object is extracted while a hand object is } \\
\text { excluded. }\end{array}$ \\
\hline 11. & $\begin{array}{l}\text { Alan } \\
\text { Lipton(1998) }\end{array}$ & $\begin{array}{l}\text { Moving Target Classification and Tracking } \\
\text { from Real-time video. }\end{array}$ & $\begin{array}{l}\text { The target can be tracked over long distances } \\
\text { and }\end{array}$ \\
\hline & & & $\begin{array}{l}\text { periods of time ( } \min 2 \text { mins. - the life span of } \\
\text { the } \\
\text { target), even as it becomes small }\end{array}$ \\
\hline 12. & $\begin{array}{|ll|}\text { Paul } & \text { Fieguth } \\
(1971) & \end{array}$ & $\begin{array}{l}\text { Color-Based Tracking of Heads and Other } \\
\text { Mobile Objects at Video Frame Rates }\end{array}$ & $\begin{array}{l}\text { the efficacy of our technique in the } \\
\text { challenging task of tracking people, } \\
\text { especially tracking } \\
\text { human heads and hands. }\end{array}$ \\
\hline 13. & Xiaolin Gu (2017) & $\begin{array}{l}\text { Appearance and Structural Motion Context } \\
\text { for } \\
\text { Multi-Target Tracking in Aerial Video }\end{array}$ & $\begin{array}{l}\text { VIVID-egg02 time is } 0.160 \text { and VIVID- } \\
\text { egs03 time is } \\
0.129\end{array}$ \\
\hline 14. & Yuan-Fang Wang & $\begin{array}{l}\text { Real-Time Multi-Person Tracking in Video } \\
\text { Surveillance }\end{array}$ & $\begin{array}{l}\text { Our system has the capability of } \\
\text { tracking multiple persons in complex scenes } \\
\text { and it } \\
\text { never } \\
\text { lost tracking. }\end{array}$ \\
\hline 15. & Jiude Li (2015) & $\begin{array}{l}\text { CAMERA-BASED HUMAN BODY } \\
\text { TRACKING USING IMPROVED CAM- } \\
\text { SHIFT ALGORITHM } \\
\end{array}$ & $\begin{array}{l}\text { Frame } 727374757677 \text {. Position } 5397139 \\
179230 \\
\text { 268. Time(s) } 4.0574 .1134 .1684 .2204 .278 \\
4.335\end{array}$ \\
\hline
\end{tabular}




\section{METHODS}

This is described in [10-13], and subtracting motion detection technology is the basis, mainly.

$D_{K}(x, y)=f_{K}(x, y)-B_{K-1}(x, y)$

$R_{K}(x, y)=\int_{0(\text { Background }), D_{k} \leq T}^{1 \text { (Foreground } D_{k}>T}$

where $\mathrm{fk}$ is the current frame image, B Background image of the current frame, and $\mathrm{T}$ is the threshold. Note that the proper threshold and background are important for the algorithm. This it can be active to achieve through iterative and empirical Values.

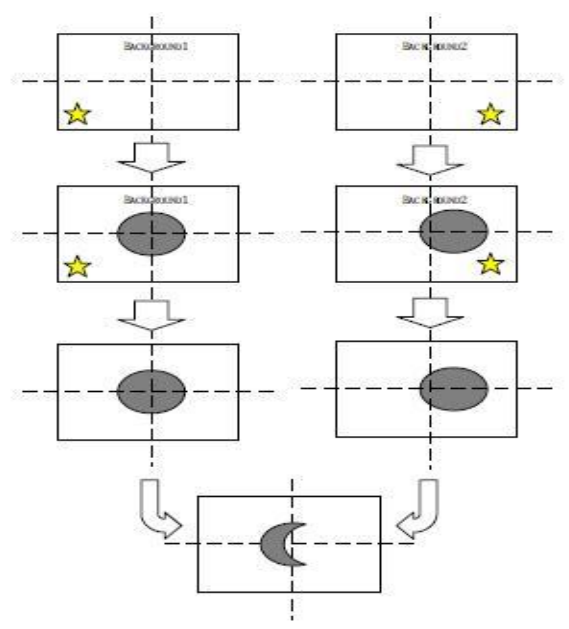

Figure 3. Principle of the motion detection with image processing.

\subsection{Mixed-Domain Tracking}

Many of optimization methods can be implemented to resolve the equation three to achieve the optimal trajectory vector $\mathrm{v} *$. However, the find-space inside deliberation is of size MT, as there are MT variable trajectory vectors considering M no of windows and slide-size know as $\mathrm{T}$. Therefore, with limited on-board resources of an underwater robot, a calculation made to determine the $\mathrm{O}$ (MT) is very expensive for its use in real time, Besides, in addition, all possible directions of movement, because the vast majority of people involved in different physical movement limited. Consequently, we adopt a search-space pruning step to eliminate these unfeasible solutions. We will discuss using the notion of hidden Markov model (HMM).

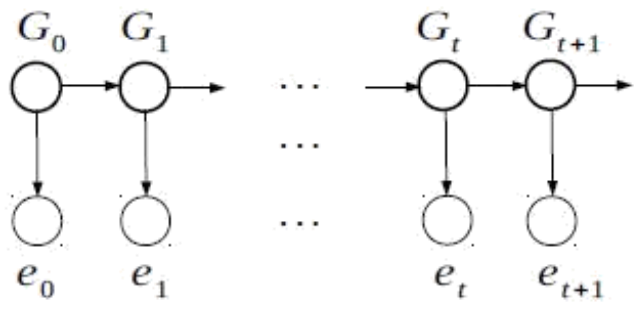

Figure 4: An HMM-based representation for the search-space of all-possible motion directions. Here, observed states (et) represent an evidence vector containing intensity values for window $w(t) i(i \in[0, M-1])$; whereas, hidden states $G t$ represent the probabilities that window $\mathrm{w}(\mathrm{t}) \mathrm{i}$ contains (partof) diver's flippers.

\subsection{Kalman filter based motion tracking.}

Let's be brief in this large, discrete filter would cover the work of a "high level" kalman filter (see previous note). After presenting This high range, which are certain equations are focused on reducing and this can be used filter. The Kalman filter calculates a method with a form of a feedback control: Filter

in some cases, the state and then receive feedback in the form of measurements (noisy). measurements. As such, the Kalman filter equations divided into two parts: firsts one is the time update equations and another one is the measurement update equations. The time update equations are depending upon for projecting move straight (in time) the current state and error covariance on the other side, as the a priori approximation error covariance reaches to zero, the gain factor $\mathrm{K}$ weights the residual less heavily. Specifically,

$\lim ?=0$

$\mathrm{G}_{8}+\rightarrow$; 
Additional method of intellectual about the weighting by $\mathrm{K}$ is that as the calculation error covariance $\mathrm{R}$ reaches to zero, the real calculation? is "trusted" more and more, while the forecast calculation $\wedge_{?}$ is reliable less and less. On the other side, as the a priori approximation error covariance ? reaches to zero the real measurement is reliable less and less, while the forecast calculation $\wedge_{?}$ is reliable more and more.

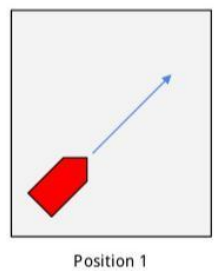

All OK

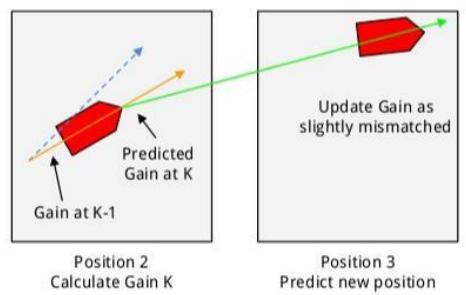

Figure 5: working of Kalman filter.

\subsection{The dynamic motion tracking}

On the basis of a random image as the implementation of the process of modeling the dynamic method. Usually, it is assumed that the image intensity derives from a Markov Random Field and, therefore, satisfies properties of locality and stationary, i.e. Every pixel is only associated to a tiny pair of neighboring pixels and dissimilar areas of the picture are perceived like. Markov random field are found inside this category. serving as a pioneer for restoring the image of plane geometry, and related activities. Start with the image of your algorithm to determine the boundaries of objects. Is to determine the next step is occluded and objects of occlusion. To this aim, a view of human psychology partially occluded objects, the so-called a modal completion method explain and studied by the Gestalt school of psychology and particularly. A certain theory presented in the recognition of the MRF model is used. What a novel confidential method to evaluate the density of the nucleus, and Markov random field (MRF), proposes a new exposure of objects. The interested video objects are 1st segmented by the user, and a nonparametric model depends on the kernel density approximation is initialized for every video object and the remaining background, respectively. One method is to identify the object to the stage, the spatial characteristics of the object, depending on the difference of the plot produced by Wavelet transformation, is a moving target is determined. The effective use of information in the temporal displacement, Markov random field prior probability model and the observation field model are established, taking benefit of Bayesian criterion, the position of object in consecutive frame is projected, and it contributes to the precise area object detection. Some author uses the markover arbitrary filed in a change way as completed in the literatures.

\subsection{Segmentation and Feature Extraction}

They will benefit from the proposed method segmentation Data dependent on time on the property. We are using fixed cameras, the environment of a camera remains relatively unchanged We have some non-material objects, including without access to education is not a "clean" image, as well as the back of the dynamic [19], verification and recovery. Once the background picture is improved, picture of the non-background objects can be divided from the background picture by differencing and thresholding [20]. The next step is to the get picture of non-background objects inside dissimilar bounding boxes. We apply the window slice technique [21] is a good time thresholded binary image is used. The first binary image, the horizontal and vertical equity is calculated up to a filter of $5 \mathrm{x}-5$. Then the gorges of the soft profiles are considered to be the boundaries of the rectangle containers holding the non-background objects and We select $\mathrm{N}$ points belonging to the medial axis of the upper body as the feature for tracking. Using multiple feature points rather than a single point [22] makes the matching of the same subject between consecutive frames more reliable.

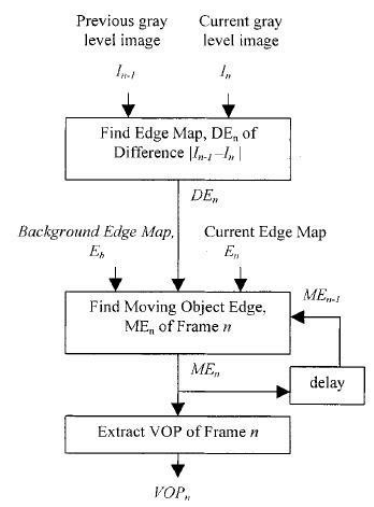

Figure 6. Block diagram of the segmentation. 
matched to the height, and the width of a subject is more probable to be done from observations. It is depending on this assumption, we permanently treat the width of a bounding box as exact information and correct the height accordingly so that all the bounding boxes for matching have the similar width and height ratio. The position of $\mathrm{N}$ feature points in the center axis of an upper body at time $t$ form a geometric feature vector

$\mathrm{Xt}=[\mathrm{xlt}, \mathrm{xZ} . \mathrm{t} ., ., \mathrm{xmt}, \ldots, \mathrm{xjvtIT}=$

$[(\mathrm{uI}, \mathrm{v} \sim)(, \sim 2,2121 ., ., .,(\mathrm{um}, \mathrm{vm}) .,-, .,(\mathrm{u} \mathrm{N}, \sim)] \mathrm{AS} \sim$

for the visual features, we use an $\mathbf{N}$ dimensional feature vector $\mathbf{Y t}=$

$\{\mathrm{ylt}, \mathrm{y} 2 \mathrm{t}, \ldots, \mathrm{y} \mathrm{N} \mathrm{t}\} \mathrm{T}$,

in which $\mathrm{y}^{\mathrm{mt}}$ is the average intensity of the neighborhood of the $\mathrm{m}^{\text {th }}$ feature points.

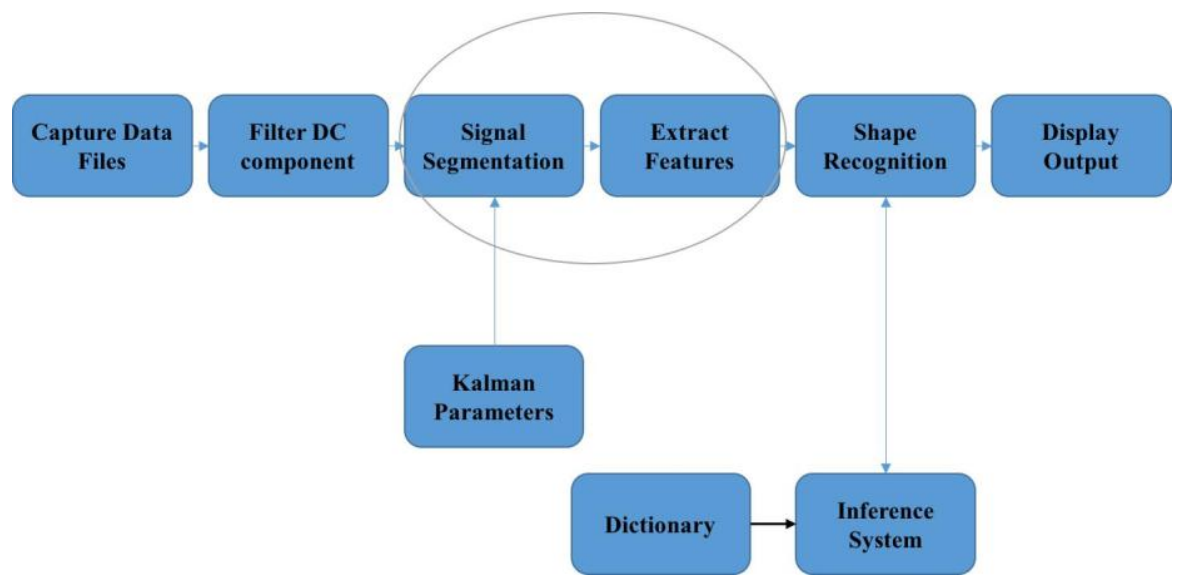

Figure 7: segmentation and feature extraction.

\subsection{Feature detection and Feature tracking}

During the feature-detection step, the image is the key element may not match the other images for the search. An overview of dissimilar detectors can be search in [23]. In order to determine the purpose of obtaining real-time performance, we chose ORB [24] for feature detection. It tremendously fast to deliver uniqueness with good invariance to deviations in viewpoint and illumination. In order to advance the robustness of the pose measurement, we split the picture into unchanging grids for feature detection and Feature tracking is an extremely important for our motion tracking system. To make a tradeoff between the robustness and efficiency in tracking, we combine the detect-then-track and the Kanade Lucas Tomasi (KLT) [25] tracker in a flexible complementary. As the name implied, the detect-then-track method is to detect features in the first image, and then, search for their corresponding matches in the following image. With respect to the KLT algorithm, the detect-then-track can provide matching features with more accurate. However, even with the ORB feature extraction, the computation cost for image stream is still a great challenge for mobile devices. Fortunately, due to the continuous image frame, the difference between neighbor images is relative small, which creates a nice preparation for the implementation of the more efficient KLT algorithm.

\section{CONCLUSION}

In this paper, an analysis is presented for different image processing techniques which can employed for object detection and tracking in videos. Various techniques of the motion tracking are discussed in this paper and various methodologies with feature extraction and comparison is also put forward. The kalman filter out of all the algorithm available have been utilized in various applications and there are numerous variants of kalman have been introduced in the past years to make kalman suited for varied applciations.

\section{REFERENCES}

[1] Weiyang Zhang, Ziqiang Cui, Dapeng Zhang, Huaxiang Wang, "Design of a Dual Camera Children Monitoring System based on Motion Tracking Technology", IEEE Instrumentation and Measurement Society prior to the acceptance and publication,978-1-5090-3596-0/17/\$31.00 (ㅇ)2017 IEEE.

[2] Md Jahidul Islam and Junaed Sattar, "Mixed-domain Biological Motion Tracking for Underwater Human-Robot Interaction", 2017 IEEE International Conference on Robotics and Automation (ICRA) Singapore, May 29 - June 3, 2017.

[3] Xiaoping Yun, Eric R. Bachmann, "Design, Implementation, and Experimental Results of a Quaternion-Based Kalman Filter for Human Body Motion Tracking", IEEE TRANSACTIONS ON ROBOTICS, VOL. 22, NO. 6, DECEMBER 2006.

[4] Reneta P. Barneva, Valentin E. Brimk, Patrick Hungt, Kamen Kanev, "MOTION TRACKING FOR GESTURE ANALYSIS IN SPORTS", Cooperative Research Project at Research Center of Biomedical Engineering with RIE Shizuoka University support by 2016.

[5] Q. Cai, J. K. Aggarwal, "Tracking Human Motion Using Multiple Cameras", 1015-4651/96, 5.00 @ 1996 IEEE Proceedings of ICPR '96.

[6] Alexandru O. Balan, Leonid Sigal, Michael J. Black, "A Quantitative Evaluation of Video-based 3D Person Tracking", Proceedings 2nd Joint IEEE International Workshop on VS-PETS, Beijing, October 15-16, 2005. 
[7] Filipe Baptista, Pedro Faria Lopes, and Pedro Santana, "MotionDesigner: Augmented Artistic Performances with Kinect-Based Human Body Motion Tracking", 2016 23॰ Encontro Portugu es de Computac, ao Gr'afica e Interac, ao (EPCGI).

[8] Dorin Comaniciu, Visvanathan Ramesh, Peter Meer, "Kernel-Based Object Tracking", IEEE TRANSACTIONS ON PATTERN ANALYSIS AND MACHINE INTELLIGENCE, VOL. 25, NO. 5, MAY 2003.

[9] Wei Fang, Lianyu Zheng, Huanjun Deng, "A motion tracking method by combining the IMU and camera in mobile devices" 2016 Tenth International Conference on Sensing Technology 978-1-5090-0795-0/15/\$31.00 @2016.

[10] Jannik Steinbring, Christian Mandery, Florian Pfaff, Florian Faion, Tamim Asfour, and Uwe D. Hanebeck, "Real-Time Whole-Body Human Motion Tracking Based on Unlabeled Markers", 2016 IEEE Intemational Conference on Multisensor Fusion and Integration for Intelligent Systems (MFI) Kongresshaus Baden-Baden, Germany, Sep. 19-21, 2016.

[11] Jan P. Vox, Frank Wallhoff, "Evaluation of Motion Tracking Methods for Therapeutic Assistance in Everyday Living Environments", 2016 IEEE Intemational Conference on Multisensor Fusion and Integration for Intelligent Systems (MFI) Kongresshaus Baden-Baden, Germany, Sep. 19-21, 2016.

[12] Cheng Yang, Gene Cheung, Vladimir Stankovic, "Estimating Heart Rate and Rhythm via 3D Motion Tracking in Depth Video", IEEE Transactions on Multimedia DOI 10.1109/TMM.2017.2672198

[13] Changick Kim and Jenq-Neng Hwang, "Fast and Automatic Video Object Segmentation and Tracking for Content-Based Applications", IEEE TRANSACTIONS ON CIRCUITS AND SYSTEMS FOR VIDEO TECHNOLOGY, VOL. 12, NO. 2, FEBRUARY 2002.

[14] Alan J. Lipton, Hironobu Fujiyoshi, Raju S. Patil, "Moving Target Classification and Tracking from Real-time Video", 0-8186-8606-5/98 \$10.00 01998 IEEE.

[15] Paul Fieguth, Demetri Terzopoulos, "Color-Based Tracking of Heads and Other Mobile Objects at Video Frame Rates", Natural Sciences and Engineering Research Council of Canada 1063-6919/97 \$10.00 01997 IEEE.

[16] Xiaolin Gu, Shilin Zhou, Lin Lei, Zhipeng Den, "Appearance and Structural Motion Context for Multi-Target Tracking in Aerial Video", 2017 2nd International Conference on Image, Vision and Computing, 978-1-5090-6238-6/17/\$31.00 @20 17 IEEE.

[17] Wei Niu, Long Jiao, Dan Han, and Yuan-Fang Wang, "Real-Time Multi-person Tracking in Video Surveillance", IEEE.

[18] Jiude Li, "RESEARCH ON CAMERA-BASED HUMAN BODY TRACKING USING IMPROVED CAM-SHIFT ALGORITHM", INTERNATIONAL JOURNAL ON SMART SENSING AND INTELLIGENT SYSTEMS VOL. 8, NO. 2, JUNE 2015.

[19] Q. Cai, A. Mitiche, and J. K. Aggarwal. Tracking human motion in an indoor environment. In 2nd Intl. Con\$ on Imge Processing, pages 215-218, Washington, D.C., October 1995.

[20] R. Polana and R. Nelson. Low level recognition of human motion (or how to get your man without finding his body parts). In Proc. of IEEE Computer Society Workshop on Motion of Non-Rigid and Articulated OS. Yalamanchili, W. N.

[21] Martin, and J. K. Aggarwal.Extraction of moving object description via differencing. CGIP, (18):188-201,1982.bjects, pages 77-82,1994.

[22] K. Jain. Fundamental of Digital Image Processing. Prentice-Hall International, Inc, 1989.

[23] S. Baker, I. Matthews, "Lucas-Kanade 20 years on : a unifying frame," International Journal of Computer Vision, 2004, pp. $221-255$.

[24] R. Siegwart, I. Nourbakhsh, D. Scaramuzza, "Introduction to autonomous mobile robots," 2nd ed, Cambridge, MA, MIT Press, 2011.

[25] E. Rublee, V. Rabaud, K. Konolige, G. Bradski, "ORB: An efficient alternative to SIFT or SURF", IEEE International Conference on Computer Vision, 2011, pp. 2564-2571. 